\title{
La cultura de la exclusión en el discurso político de los universitarios colombianos
}

... en unos sitios los genéticamente incapaces son los negros, en otros los indios, los gitanos o los esquimales y en casi todos los hijos de los pobres.

Jairo Eduardo Soto Molina

F: Savater

\author{
languagecircle.re@ hotmail.com \\ Universidad del Atlántico \\ Milys Karina Rodelo Molina \\ milys422@hotmail.com \\ Universidad del Atlántico
}

\section{Resumen}

El propósito de la investigación fue identificar los factores que determinan la exclusión en la educación superior, analizar posibles causas y proponer formas para disminuir estas conductas colectivas y garantizar los derechos humanos fundamentales y el bienestar social de los sujetos en edad universitaria. Los informantes clave fueron 166 personas, 30 docentes, 22 egresados y 114 estudiantes, quienes permitieron identificar los elementos del conflicto para precisar formas de solución a través de una perspectiva crítica que develaron las prácticas de exclusión más frecuentes entre los estudiantes. Existen otros factores asociados a la fragmentación curricular, e inciden en la exclusión educativa evitando formas homogéneas entre la población universitaria. Otro de los factores, se relacionan con el estatus socioeconómico, cultural, la etnia, el género y más aún el factor ideológico, los cuales influyen en el desarrollo educativo, las tasas de fracaso y retención, dificultando así, una escolarización igualitaria y de calidad. En síntesis, la exclusión educativa ha sido observada desde el punto de vista de factores diversos: del acceso, la financiación de la educación, las características del currículo, la pedagogía o las relaciones de apoyo entre los diferentes agentes del sistema educativo.

\section{Palabras clave}

Educación inclusiva; exclusión; formación política; tolerancia alteridad.

Recibido 15/01/2020-Aceptado 21/02/2020 


\title{
The culture of exclusion in the political discourse of Colombian university students
}

\begin{abstract}
The purpose of the research was to identify the factors that determine exclusion in higher education, analyze possible causes and propose ways to reduce these collective behaviors and guarantee the fundamental human rights and social well-being of subjects of university age. The key informants were 166 people, 30 teachers, 22 graduates and 114 students, who made it possible to identify the elements of the conflict in order to define forms of solution through a critical perspective that revealed the most frequent exclusion practices among students. There are other factors associated with curricular fragmentation, and they affect educational exclusion, avoiding homogeneous forms among the university population. Another factor is related to socioeconomic, cultural status, ethnicity, gender, and even more so the ideological factor, which influence educational development, failure and retention rates, thus hindering equal and quality schooling. . In summary, educational exclusion has been observed from the point of view of various factors: access, financing of education, characteristics of the curriculum, pedagogy or support relationships between the different agents of the educational system.
\end{abstract}

\section{Keywords}

Inclusive education, exclusion, political formation, otherness tolerance. 


\section{A cultura da exclusão no discurso político de universitários colombianos}

\section{Resumo}

O objetivo da pesquisa foi identificar os fatores que determinam a exclusão no ensino superior, analisar possíveis causas e propor formas de diminuir esses comportamentos coletivos e garantir os direitos humanos fundamentais e o bem-estar social dos sujeitos em idade universitária. Os informantes-chave foram 166 pessoas, 30 professores, 22 graduados e 114 alunos, que possibilitaram identificar os elementos do conflito para definir formas de solução por uma perspectiva crítica que revelou as práticas de exclusão mais freqüentes entre os alunos. Existem outros fatores associados à fragmentação curricular, que afetam a exclusão educacional, evitando formas homogêneas entre a população universitária. Outro fator está relacionado ao nível socioeconômico, cultural, étnico, de gênero e, mais ainda, ao fator ideológico, que influencia as taxas de desenvolvimento educacional, reprovação e retenção, dificultando a escolaridade igual e de qualidade. . Em resumo, a exclusão educacional foi observada do ponto de vista de vários fatores: acesso, financiamento da educação, características do currículo, pedagogia ou relações de apoio entre os diferentes agentes do sistema educacional.

\section{Palavras-chave}

Educação inclusiva, exclusão, formação política, tolerância à alteridade. 


\section{Introducción}

La universidad es un contexto en el cual concurren fuerzas centrípetas (aquellas que están dirigidas a su interior) y fuerzas centrífugas (aquellas que están dirigidas hacia el exterior), las cuales, en momentos determinados de la historia de las instituciones universitarias, se han impuesto unas sobre otras. Durante las décadas de los años 60s y 70s se concibió la educación como un factor de calidad de vida en general, por su fuerza transformadora al interior de la sociedad y las posibilidades de ascenso y desarrollo social que brindaba. A partir de la década de los 80 s se considera a la educación como conservadora del statu quo.

Actualmente, no se discute la coexistencia de estos dos factores en la educación: transformación y conservación. Por consiguiente, la educación en y para la diversidad se propone potenciar el aspecto transformador, en el sentido de hacer efectiva la igualdad de oportunidades. Lo cual no significa dar a todos lo mismo, sino a cada uno lo que corresponde. Por lo tanto, el enfoque de una educación en y para la diversidad se enmarca en la concepción de igualdad y equidad. Esta concepción sostiene que no todos los estudiantes universitarios llegan a la institución en las mismas condiciones.

Ante tal situación, es indiscutible que las diferencias y jerarquías sociales existen y se manifiestan a la hora del acceso a la educación superior, al trabajo, la vivienda, la salud y la justicia. Se observa que la universidad las reconoce y legitima en los mismos términos; logrando establecerlas como diferencias que son interpretadas jerárquicamente desde la lógica universitaria. De este modo, el fracaso en los estudios de nivel superior es explicado tanto como déficit sociocultural, como por insuficiencias de índole "personal" en determinadas capacidades. En atención a esas diferencias, se generan dispositivos de pedagogía compensatoria, muchos de los cuales, en programas especiales, dan una sensación de minusvalía en su tratamiento.

Desde este contexto, surgen programas con buenos propósitos de "atender mejor", partiendo del déficit habitual (de lo que falta), con un enfoque segregacionista. Otro aspecto del funcionamiento de este enfoque es el carácter “compensador”, siendo este la primacía del enfoque asistencialista, que desplaza la atención de lo pedagógico para dar énfasis exclusivamente en el problema social de los estudiantes. 
En cambio, si se concibe la educación con sus posibilidades transformadoras, -el hecho que los estudiantes no lleguen a la universidad en las mismas condiciones-, es interpretado como diversidad y no como desigualdad. Esto supone, necesariamente emplear otra lógica y seguir otra línea de pensamiento que conduce a otros resultados. La universidad en y para la diversidad tiende a integrar las diferencias como lo genuino de una sociedad que se pretenda pluralista, democrática y socializante, "la integración escolar y social como ruptura epistemológica e ideológica ha de ser la nueva energía que posibilite ese cambio cualitativo que mejore la calidad de vida de los ciudadanos [...]" (López, 1991, p.25). En otras palabras, se requiere asumir retos y desafíos atendiendo a las necesidades de la población objeto de estudio, para generar y sostener condiciones y estrategias que proporcionen a cada estudiante la atención pedagógica que necesite, tanto en lo personal como en lo experiencial y sociocultural.

En la búsqueda de antecedentes del tema, se revisaron numerosos libros y artículos publicados referidos a investigaciones sobre educación inclusiva. Previamente, el libro de Forlin \& Loreman (2014) "Measuring Inclusive Education", ilustra desde la perspectiva crítica, cómo se puede medir de manera efectiva la inclusión. El texto, considera los problemas asociados con la inclusión y proporciona ejemplos de buenas prácticas basadas en evidencia y modelos de medidas efectivas. Sus autores son investigadores o profesionales experimentados y brindan información altamente relevante para la recolección de información propuesta en el estudio, el cual es de valor para todo el personal comprometido en los esfuerzos de medir la educación inclusiva en nuestro contexto. Principalmente, la citada investigación se enfoca en medir la educación inclusiva desde distintas perspectivas. Se fundamenta en conceptualizaciones internacionales de educación inclusiva y las formas en que los diferentes sistemas miden su impacto y efectividad.

En la primera instancia, de presentan temas generales asociados con la evaluación para, de y en el aprendizaje en aulas inclusivas; dilemas éticos, que miden la implementación de políticas inclusivas; las cuales pretenden identificar y eliminar barreras a la inclusión; proveer dotación de recursos y financiación; y evaluar la preparación efectiva del maestro para la inclusión, proporcionando la estructura para la discusión. 
Otro de los principales hallazgos, se presenta una revisión actual y profunda de la literatura sobre el desarrollo de indicadores internacionales para medir la educación inclusiva. Se estudian ejemplos de modelos empleados actualmente para enmarcar una evaluación de la práctica inclusiva. Estos ilustran estrategias de medición operativas para identificar prácticas inclusivas en todos los niveles de administración, desde el nivel macrocurricular en instancias gubernamentales, hasta el nivel microcurricular en las aulas de clase, con el propósito de reconocer resultados positivos alcanzados por todos los involucrados.

El segundo antecedente revisado, fue el capítulo del libro publicado por Ebersold, S., \& Meijer, C. (2016), donde destaca aspectos que ocupan un lugar relevante para repensar los mecanismos de asignación de recursos, el tema del empoderamiento y la forma en que estas estrategias de financiación apoyan la educación inclusiva. El estudio, se enfoca en factores críticos de financiamiento que apoyan el derecho a la educación, como se describe en el Artículo 24 de la Convención de las Naciones Unidas sobre los Derechos de las Personas con Discapacidad (UNCRPD), (2006), en un contexto de restricciones financieras y detalla la brecha de inequidad entre la política pública y la práctica en relación con las prioridades y objetivos políticos nacionales y europeos. Asimismo, resalta referentes previos de modos de financiación, en investigaciones realizadas por la Agencia Europea y en el marco conceptual desarrollado dentro de un nuevo estudio sobre políticas y prácticas actuales en este campo.

El tercer trabajo revisado, es el de Porter (2011) Titulado "Diversity, Equity and Inclusion" en su capítulo "Exploring Inclusive Educational Practices Through Professional Inquiry”, trata sobre los niños y los adultos que se preocupan por ellos. En el estudio, se cuenta historias reales de maestros, directores, padres y personas que trabajan con niños con diversas necesidades que dificultan su traslado a la escuela comunitaria. Y, sí, deberíamos preocuparnos por qué sucede realmente en esas aulas, en las mismas clases en que están esos niños. En otras palabras, están "incluidos" y esta acción simple se denomina "educación inclusiva".

Por último, el trabajo desarrollado por los docentes de la Universidad del Atlántico, De la Hoz \& Mercado (2019), quienes muestran en su estudio como esta institución ha sido líder en el conocimiento, promoviendo el desarrollo y transformación de la Región Caribe. 
Del mismo modo, desde el 2007 ha venido impulsando la inclusión de estudiantes con discapacidad en sus programas. Para tales propósitos, el Proyecto Diverser se creó con la finalidad de apoyar la educación inclusiva en primera instancia de personas con discapacidad auditiva y paulatinamente fueron ingresando otros estudiantes con discapacidad visual y física.

La Universidad del Atlántico ha sido la segunda universidad en continuar este proyecto que inició en la Universidad Pedagógica Nacional, un 28 de septiembre de 2009, fecha en la cual se inaugura la estrategia en el ámbito universitario. Su actual creadora Oris Mercado, hizo un recorrido por las universidades del país y advirtió la ausencia de este programa en la Universidad del Atlántico, de allí su creación. Al principio, solo se atendió la población con discapacidad y a partir de 2015 a población diversa de todo tipo. De esta manera, se comenzaron a crear las condiciones que han contribuido a que esta población mejore su calidad de vida, permitiendo condiciones óptimas y oportunidades de empleo e inclusión social (Mercado \& De la Hoz, 2019).

De otra parte, se hizo una indagación al respecto y contrastando con los lineamientos del Ministerio de Educación Nacional (MEN) y la política inclusiva propuesta por la Organización de las Naciones Unidas para la Educación, la Ciencia y la Cultura (UNESCO), se desarrollaron actividades y experiencias significativas las cuales fueron sistematizadas; al final del proceso de sistematización, se establecen diversas situaciones de desigualdad económica, cultural, política y afectiva, las cuales son generadas entre otras circunstancias por desigualdades en la misma política educativa e institucional.

Esta mirada multidimensional, permitió clasificar los factores que inciden en la exclusión educativa, el cual es uno de los valores añadidos de este informe, el cual permitió diagnosticar la situación de la institución intervenida y ordenar las propuestas de posible solución. Para ello, se analizan las distintas situaciones discriminatorias a las que se enfrenta el estudiante universitario, las cuales en la mayoría de los casos genera procesos de desvinculación, deserción, fracaso y abandono de los estudios. 


\section{Una universidad integradora, inclusiva y comprensiva}

En esta concepción, la universidad es un espacio preferencial para desarrollar actitudes individuales y sociales que generen cambios significativos en las personas. La universidad integradora, inclusiva o comprensiva está sustentada en una pedagogía que se opone a la sección jerárquica y propone una voluntad explicita de desarrollar a los estudiantes en un contexto universitario respetuoso de la diversidad personal y colectiva.

Atender a los jóvenes universitarios adecuadamente respetando su modo de ser (diversidad) no es una ilusión teórica, es también una práctica necesaria. Lo que demanda la continuación de un proceso complejo y cargado de dificultades, no solo recursos y medios, sino un cambio en las convicciones culturales, sociales, y educativas en toda la sociedad. La cultura de la diversidad requiere una renovación de los enfoques de la enseñanza en las escuelas y universidades, propiciando una visión crítica de la educación, y no meramente reformista de las estructuras institucionales.

Es así, como la atención a la diversidad es considerada uno de los pilares básicos sobre los que se fundamenta todo cambio educativo que proponga desarrollar modalidades de actuación comprensivas y respetuosas de identidades personales o grupales. Aun cuando este proceso resulte complejo, porque se confronta contra la universidad selectiva dominante, no es imposible. Lo que requiere plantear un reto político y educativo, debido a que las cuestiones ideológicas están siempre presentes en estas temáticas. Es una falacia hablar de neutralidad cuando se hace referencia a la diversidad: cualquier opción que se tome está marcada y condicionada por valores y contextos socioeducativos. En síntesis, la diversidad es un rasgo común y objetivo de toda la dinámica social (Soto, 2015).

Ante este hecho de interés mundial, la Organización de Naciones Unidas para la Educación, la Ciencia y la Cultura (UNESCO), considera que la "educación inclusiva y de calidad se basa en el derecho de los alumnos a recibir una educación de calidad que satisfaga sus necesidades básicas de aprendizaje y enriquezca sus vidas”. Asimismo, en lo relacionado con la inclusión y equidad, que propone el programa DiverSer de la Universidad del Atlántico, que asume como principios transversales y conductores para la transformación del sistema educativo, "garante de la igualdad de oportunidades, la no discriminación, la calidad con equidad, el reconocimiento de capacidades, ritmos, estilos de aprendizaje”, para lo cual 
todo lo que contribuya a su operatividad (presupuesto, gestión educativa, becas, evaluación, planes, métodos, maestros, autoridades y familia) guiará estos principios.

Para lograr la inclusión y equidad en educación superior se propone una intervención curricular. Al ser adaptable, debe ser flexible, así la universidad o los docentes pueden desarrollar, contextualizar los temas y atender las especificidades de los estudiantes. Se busca que la educación que se imparta en nuestra institución debe valorar la riqueza de nuestra sociedad pluricultural y metalingüística. Brindando además todas las facilidades a las poblaciones que presentan algunas particularidades especiales como los sordos, ciegos, y personas con discapacidades de movilidad.

Las acciones expresadas, operaran con acompañamiento técnico y supervisión, atendiendo que todo estudiante, aun estando en el nivel de educación superior, merece una consideración desde diferentes perspectivas. La ambigüedad de la palabra fracaso que se presenta como aparentemente neutra, tiene la velada intención de imputarle la responsabilidad del fracaso al estudiante.

\section{Políticas nacionales de educación inclusiva}

El Ministerio de Educación Nacional, ha establecido lineamientos de Educación Inclusiva con los cuales busca incentivar en las IES acciones y estrategias para el fortalecimiento del enfoque diferencial en el acceso, permanencia y condiciones de calidad de las poblaciones de especial protección constitucional (Población Víctima, Población con discapacidad, Grupos étnicos - indígenas, comunidades negras, Rom ${ }^{1}$ )

Según la política de estado, se plantea una educación inclusiva como estrategia central para la atención a la diversidad, una inclusión que trasciende la dicotomía o tradicional asociado al concepto de exclusión que permite pensar un modelo educativo abierto y generoso atendiendo a la diversidad como una característica inherente no solo al ser humano sino a la vida. Con esta apuesta se propone avanzar en el cierre de brechas de inequidad, mediante el pleno ejercicio de derechos y el acceso a las oportunidades a través de medidas estructurales de política púbica que contribuyan a la consolidación de una paz estable y

\footnotetext{
1. La vida del Pueblo Rom en Colombia todavía se mantiene, pese al paso del tiempo. La comunidad gitana o Pueblo Rom es un grupo étnico que llegó a América Latina desde el tiempo de la Colonia. Hoy, aproximadamente 4.830 de ellos están radicados en varias ciudades de Colombia; Población de Frontera.
} 
duradera. Señala González que, "en los últimos años el concepto de inclusión ha designado un terreno fundamentalmente disputado por una multiplicidad de disciplinas académicas, discursos, concepciones políticas y corrientes críticas más prominentes de nuestra época” (2019:29).

En este sentido, los lineamientos de educación inclusiva, publicados en el 2014 se han convertido en un documento pionero en América Latina, que responde al interés por las instituciones de educación superior de nuestro país para desarrollar este modelo educativo como núcleo vital de una sociedad que busca la paz desde la reparación y la reconciliación. Desde estas acciones, se abre el camino para lograr que la educación superior de Colombia potencie y valore la diversidad protegiendo las particularidades, el respeto a ser diferente y a la participación de la comunidad dentro de una estructura intercultural.

Las orientaciones de política frente a la educación inclusiva cuentan además con un componente transversal frente al enfoque de identidad de género, que complementan las existentes políticas y propuesta de acciones, con el ánimo de brindar mayores elementos a las IES, con lo que se ha desarrollado un índice de Inclusión como una herramienta para afrontar el reto de alcanzar un sistema de educación superior inclusivo. Esto permite reconocer las condiciones actuales para la atención a la diversidad, así como también analizar sus fortalezas y oportunidades de mejora como proceso que contribuye al aprendizaje, la participación y la convivencia de la comunidad.

Adicionalmente, el Ministerio de Educación Nacional en el marco de sus funciones de fomento a la Educación Superior, ha puesto en marcha acciones específicas con poblaciones que históricamente han tenido mayores barreras de acceso, permanencia y graduación al sistema. Por ejemplo, los pueblos indígenas cuentan con el Decreto 1953 de 2014, mediante el cual se avanza en la posibilidad crear sus instituciones educativas y requisitos de organización y proyectos educativos propios mediante un régimen transitorio, como personería jurídica y reconocimiento de sus procesos de educación superior. Asimismo, se cuenta con acciones articuladas con ICETEX y Ministerio del Interior, para la financiación del acceso y permanencia de los estudiantes indígenas a la educación superior. 
Ante la población con discapacidad, se ha constituido una alianza denominada, "Apoyo financiero para estudiantes con discapacidad en Educación Superior", entre el MEN, ICETEX y La Fundación Saldarriaga Concha, por el cual personas en situación de discapacidad pueden recibir apoyos en la matrícula o sostenimiento de su programa. En lo relacionado a la población víctima del conflicto armado, en el año 2013 se constituyó el Fondo de Reparación para el Acceso, Permanencia y Graduación de la Población Víctima del Conflicto Armado en Colombia, considerando lo estipulado en el artículo $3^{\circ}$ de la Ley 1448, para gestionar apoyo a la población que no cuente con recursos económicos para acceder a la educación superior y que se destaquen por su desempeño académico.

En beneficio a las Comunidades Negras, Afrocolombianas, Palenqueras y Raizales, se han definido acciones para el acceso y permanencia de estas poblaciones a la Educación Superior, liderado por Ministerio del Interior y el ICETEX, entre estas se destacan la financiación, creación y puesta en marcha del Fondo de Comunidades Negras, el cual es un fondo destinado a facilitar el acceso, permanencia y graduación de estudiantes garantizando el derecho a la igualdad de oportunidades con relación con el resto de la sociedad colombiana.

Para el caso de la población Rom, se ha incluido dentro de las estrategias de financiación un fondo para promover el acceso, permanencia y graduación de la población en la educación superior, mediante la financiación de la matrícula y un subsidio de sostenimiento. Sin embargo, estos aspectos no se deben abordar aisladamente sino en un conjunto de subsistemas. El enfoque sistémico sostiene que el comportamiento humano está determinado por la estructura del sistema a la que pertenece el sujeto y su posición dentro de éste. Si el sistema cambia es factible que también cambie el sujeto. De la misma manera en que el sujeto conforma al sistema, del mismo modo el sistema se encuentra determinado por el individuo.

En efecto, la actual problemática implica la reflexión del lenguaje sobre sí mismo: es ver al ser humano como un producto del lenguaje social generador de nuevas realidades. Su mal uso indiscriminadamente conduce a momentos aporéticos. En conclusión, estas acciones son producto de los razonamientos en que se generan contradicciones o paradojas irresolubles; momentos en que las aporías se presentan como dificultades lógicas casi siempre de índole especulativo, cargadas de juicios, producto de la no aceptación de las diferencias o al no reconocimiento de la cultura de las otras personas en su interacción con otros. Es un problema de desconocimiento del valor de la alteridad y la otredad (Soto, 2017). 


\section{Materiales y métodos}

Se implementaron procedimientos de análisis documental para revisar y analizar los distintos factores que determinan la exclusión y sus causas. Posteriormente, se recogió la información a través de cuestionarios con preguntas de selección múltiple, dirigidas a 166 informantes, entre los que estuvieron 30 docentes, 22 egresados y 114 estudiantes, quienes aportaron información suficiente para identificar la problemática, hechos de interés y posteriormente ahondar en ellos a través de foros de discusión como medios para precisar formas de coadyuvar a la solución a través de una perspectiva crítica frente a las prácticas de exclusión más frecuentes entre los estudiantes universitarios.

\section{Resultados y discusión}

Desde el análisis relativo a la primera de las ocho preguntas, se destacó la percepción de universidad como una institución que:

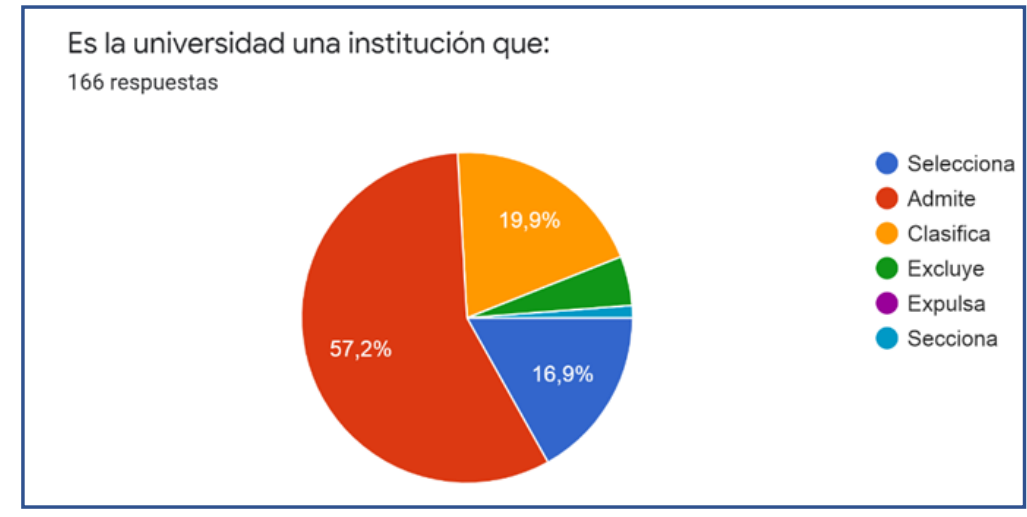

Figura1. Factores de exclusión. Elaboración propia (2020).

a. Selecciona $16,9 \%$. Podría decirse que las jerarquías vigentes en la sociedad global se vinculan con modelos de excelencia, algunos de los cuales se reeditan en la universidad. La universidad no genera las jerarquías de excelencia ni son de patrimonio exclusivo. También es cierto que no todas las jerarquías sociales de excelencia tienen su correlato en la universidad. Pero es evidente que las jerarquías universitarias no están aisladas de las significaciones sociales. Por ejemplo, el dominio de la lengua de Occidente ocupa un lugar privilegiado en todos los ámbitos tanto de la comunicación oral como la escrita. En cuanto a ello, 
(...) los vínculos existentes entre las jerarquías escolares y otras jerarquías de excelencia son tanto más explicables en la medida en que se pretende que la enseñanza constituya una preparación para la vida: en este sentido, las clasificaciones escolares no son sino la prefiguración de jerarquías vigentes en la sociedad global, en virtud de modelos de excelencia que reciben una valoración suficiente como para ocupar un espacio en el currículo (López, 1991). (p. 25).

El modelo selectivo ubica a los estudiantes mediante parámetros teóricos, estadísticos, organizativos y, por ende, artificiales y externos. No se considera el desarrollo particular y peculiar de los grupos ni las personas. Se pretende que todos los estudiantes progresen al mismo ritmo en el discurso en el que se reconoce la diversidad de los ritmos de aprendizaje.

En el modelo selectivo se valoran más las capacidades que los procesos, los agrupamientos homogéneos que los heterogéneos. La competitividad que la cooperación, el individualismo que el aprendizaje solidario o cooperativo, los modelos cerrados, rígidos, inflexibles que los proyectos educativos abiertos, comprensivos y transformadores. La concepción de educación selectiva se apoya en modelos tecnicistas, no en modelos holísticos y ecológicos. Se pone el énfasis en enseñar contenidos académicos como medio de desarrollar las habilidades y las destrezas y no contenidos culturales y vivenciales de interés real para los estudiantes. Este modelo privilegia además la evaluación por resultados y no por procesos, con un criterio supuestamente objetivo.

b. Admite 57\%. La admisión es una parte del modelo selectivo, otro efecto del carácter clasificatorio del sistema educativo en su segmentación que lleva a dar educación de pobres para pobres y educación de ricos para ricos a partir de parámetros que atienden a los sectores más necesitados. En ese sentido el programa "Jóvenes en acción” es una ayuda no muy definitiva, pero en algo sirve como aliciente para darle continuidad a un estudiante de tiempo completo en su ejercicio académico al interior de la universidad.

c. Clasifica, 19,9\%. La clasificación es un proceso selectivo mucho más riguroso posterior a la selección o admisión. Se fragmenta mucho más el universo de la población estudiantil por programas atendiendo unas variables que operan en los exámenes de estado. 
d. Excluye, 9\%. La segregación a clases especiales; se dirigen a estudiantes que se consideran inadaptados para la escolaridad común, por su retardo o lentificación en el desarrollo cognitivo o ritmo de aprendizaje, como trastornos conductuales.

e. Expulsa, 0\%. Nada que decir.

f. Direcciona, 2.2\%. De una u otra manera se haya una directriz en tiempos de confusión a un tratamiento pedagógico, médico o de cualquier otra índole.

A la pregunta, la inclusión se refiere a, estos fueron las respuestas:

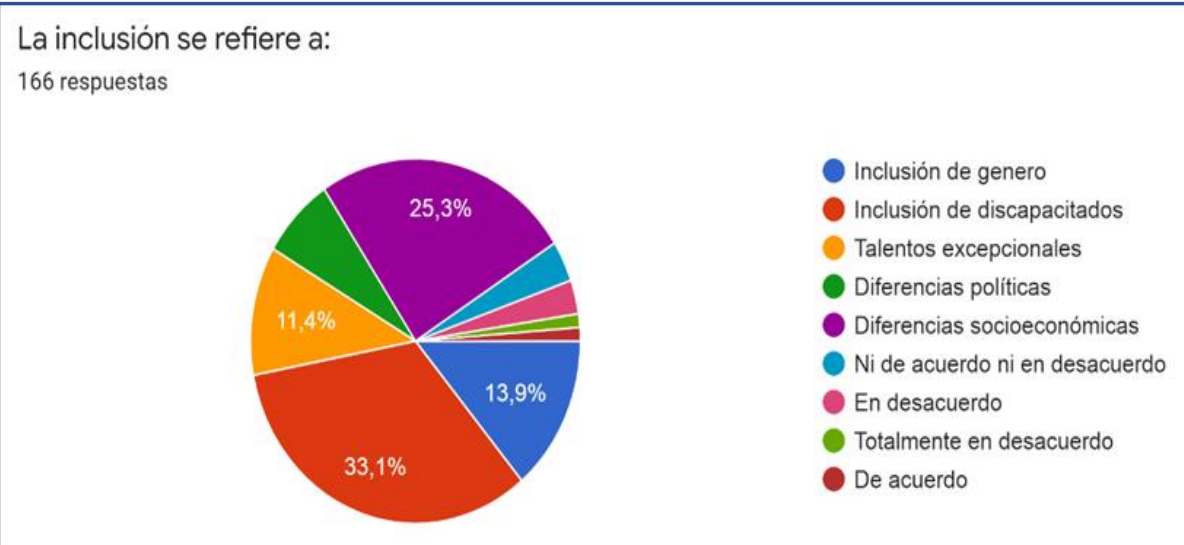

Figura 2. Factores de inclusión.

Elaboración propia (2020).

Se actúa desde el convencimiento de que la diversidad constituye algo natural e inherente a cualquier grupo humano, por lo que todo el estudiantado es diverso desde esa perspectiva haciendo referencia en concreto a la diversidad cultural, este estilo educativo de enfocar el trabajo en el aula reconoce que esa diversidad está vinculada a los siguientes factores:

La Universidad del Atlántico a través de la Vicerrectoría de Bienestar Universitario tiene claros propósitos de esta política de inclusión de género en lo concerniente a establecer una clara visión, dirección, un lenguaje común y mensaje coherente para poner en práctica y comunicar el compromiso institucional con la promoción de la igualdad de género, los derechos de las jóvenes y mujeres e inclusión para todo el personal docente y de trabajadoras, a todos los niveles. 
Muy a pesar de que se muestra un porcentaje que no es de los más altos, en lo concerniente a la inclusión de género con un 13.9\%, en la institución se han diseñado, implementado, monitoreado y evaluado los programas de género e inclusivos, y de calidad. Se han implementado estrategias de largo plazo para el cambio de las normas sociales para poner fin a la injusticia, desafiar las relaciones desiguales de poder y abordar las causas subyacentes de la posible discriminación.

Se integran medidas de igualdad de género e inclusión que alinean y complementan lo establecido en los estatutos generales y específicos de la institución. La institución no tolera las prácticas que resulten en la discriminación de género, exclusión o desigualdad de género u otras formas de identidad. En la institución se cuenta con organizaciones y grupos de investigación, los cuales fortalecen y extienden su base de colaboración con énfasis especial en los grupos representativos como los movimientos de derechos de mujeres y niñas, organizaciones de personas con discapacidad, movimientos de pueblos indígenas, afros y redes LGTBI, entre otros;

La inclusión de discapacitados es relativamente alta 33.1\%, seguido por el de diferencias socioeconómicas $(25,3 \%)$, lo que indica que, en la Universidad del Atlántico, no se nota la diferencia de estratos socioeconómicos al igual que la de los talentos excepcionales, $11.4 \%$, en la cual se evidencia su inclusión

En lo relativo a la exclusión por razones políticas o ideológicas se puede apreciar lo siguiente:

Los grupos políticos de diferentes matices conviven en el respeto de sus diferencias en la Udela 166 respuestas

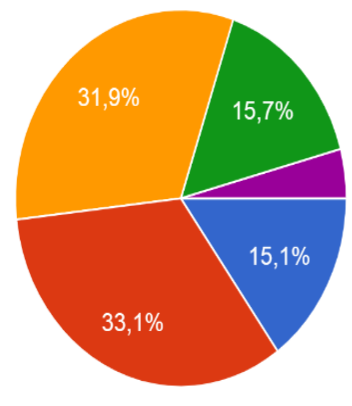

Figura 3. Aspectos de exclusión política Elaboración propia (2020). 
Una de las características de la comunidad uniatlanticense ha sido la intolerancia política hacia los grupos opositores, en ello, se debe precisar que esto no es como en décadas pasadas que había diferencias ideológicas y mucha más tolerancia en los aspectos de convivencia. En este aspecto se ha retrocedido, lamentablemente los porcentajes de los encuestados lo señalan claramente, con un $33.1 \%$ en desacuerdo y un $31.1 \%$ ni de acuerdo, ni en desacuerdo; frente a un $15,1 \%$ totalmente en desacuerdo y otro porcentaje similar, de un $15,7 \%$ de acuerdo.

Esta actitud de intolerancia ha alcanzado su clímax en el espacio universitario en las dos últimas décadas en que la universidad ha llegado a convertirse en un círculo político más de la ciudad y el departamento. En efecto, ya en esos cenáculos, como las asambleas estudiantiles, no se juzga a los estamentos universitarios al interior del Alma Mater por sus cualidades morales, intelectuales, o académicas, sino simplemente por sus creencias ideológicas. Quien no coincida con sus ideas es automáticamente descalificado y pasa a ser ipso facto un enemigo de ese sector. Por lo tanto, hay que removerlo de su cargo. En general las universidades públicas del país se han convertido en focos de intolerancia política e ideológica en la que satanizan y hasta persiguen a quienes no comulgan con las propuestas "progre" de los grupos dominantes, aunque no sean mayoritarios, caso que se presenta tanto a nivel estudiantil como en algunos miembros de algunas administraciones de las respectivas IES públicas.

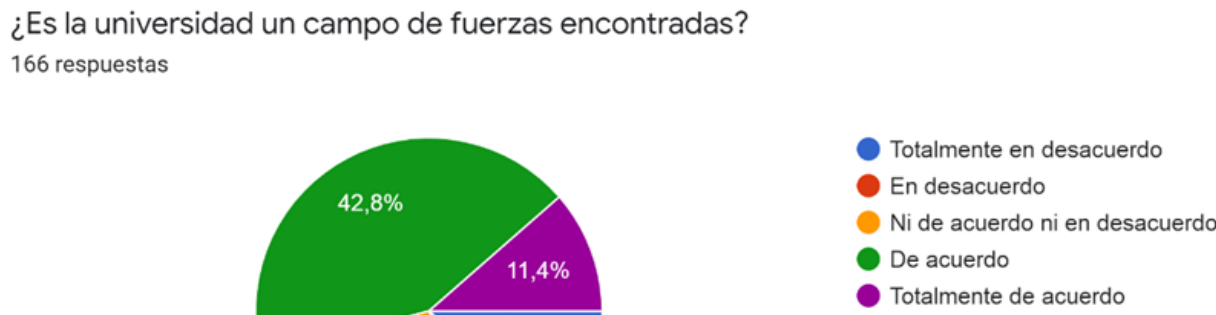

Totalmente en desacuerdo

En desacuerdo

Ni de acuerdo ni en desacuerdo

De acuerdo

Totalmente de acuerdo

Figura 4. Elementos de contradicción en el ejercicio político estudiantil Elaboración propia (2020). 
Las anteriores consideraciones se ratifican en la anterior gráfica, en la cual un $42.8 \%$ afirma que la Universidad del Atlántico es un campo de fuerzas encontradas, con lo que un $11,4 \%$ manifiesta estar totalmente de acuerdo; en tanto un $28.9 \%$ dice no estar ni de acuerdo o en desacuerdo y sólo un 11,4\% dice estar en total desacuerdo.

La política de la tolerancia debería ser una alternativa ante sociedades polarizadas, confrontadas y marcadas por el antagonismo y que han degenerado en violencia de todo tipo, con las características de la colombiana. Si la intolerancia parece imponerse en la medida en que triunfa el poder de la coacción, la tolerancia puede conducir a un horizonte en el que predomine la razón civilizada. Por consiguiente, este campo de fuerzas encontradas debe aminorarse o atenuarse mediante la expedición de nuevos estatutos que garanticen la paz institucional, que le permita una mejor gobernanza a las futuras administraciones de esta institución de educación superior.

En cuanto a la posición de la administración de la Universidad del Atlántico frente a estas diferencias políticas o de concepción ideológica los estudiantes se expresan así:

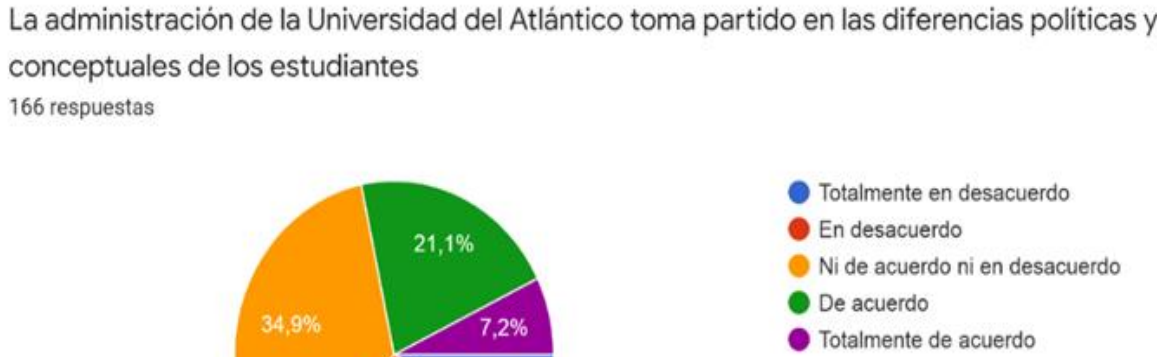

Totalmente en desacuerdo

- En desacuerdo

Ni de acuerdo ni en desacuerdo

De acuerdo

Totalmente de acuerdo

Figura 5. Elementos incidentes de contradicción política desde la Administración Elaboración propia (2020).

Los porcentajes muestran claramente con un 28,9\%, que salvo algunos casos excepcionales la administración de la universidad ha tomado partido en estos tipos de enfrentamientos ideológicos, pero se cuestiona la contratación de activistas tanto de derecha como de izquierda. Esto no es bien visto porque se relaciona como chantaje o negociación o contratación de fuerzas aliadas según sea el caso. 
En relación con las diferencias religiosas, al interior de la institución universitaria se expresa:

Existe respeto por las diferencias religiosas en la Universidad del atlántico

166 respuestas

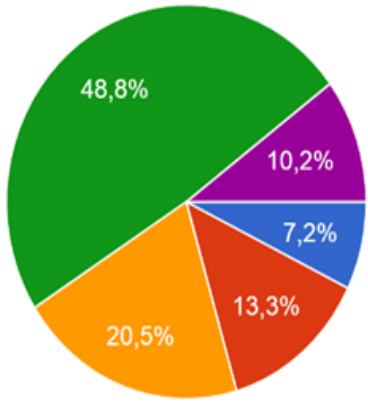

Totalmente en desacuerdo

En desacuerdo

Ni de acuerdo ni en desacuerdo

De acuerdo

Totalmente de acuerdo

Figura 6. Factores de tolerancia de grupos religiosos.

Elaboración propia (2020).

El elevado porcentaje de la opción "De acuerdo", con un 48,8\%, declarando respeto por las diferencias religiosas al interior del Alma Mater; un 19,2\% totalmente de acuerdo con esta afirmación; y un 20,5\% ni de acuerdo ni en desacuerdo. De igual modo se señala que los profesores son respetuosos de las anteriores diferencias tanto políticas e ideológicas como las religiosas con contundentes porcentajes: así por ejemplo hay un $12 \%$ totalmente de acuerdo, un 34,9\% de acuerdo y un 33,1\% que, ni de acuerdo ni en desacuerdo frente a la afirmación anterior.

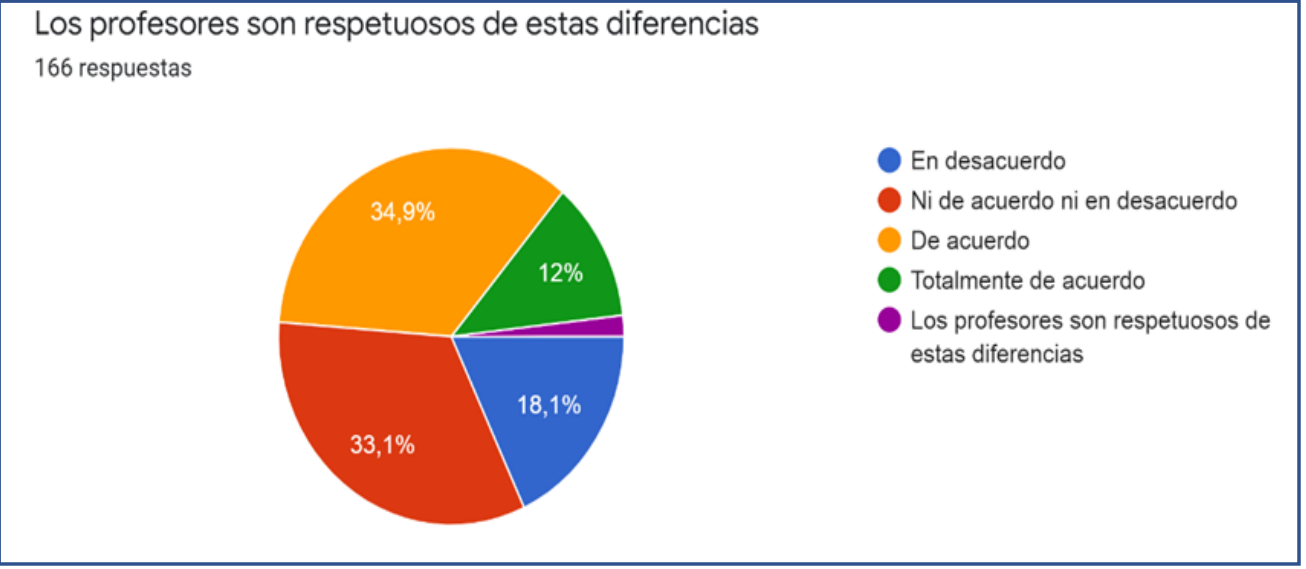

Figura 7. Factores que inciden en las diferencias Elaboración propia (2020). 
Frente a las posibles soluciones a los factores de exclusión últimamente presentados los estudiantes señalan lo detallado a continuación:

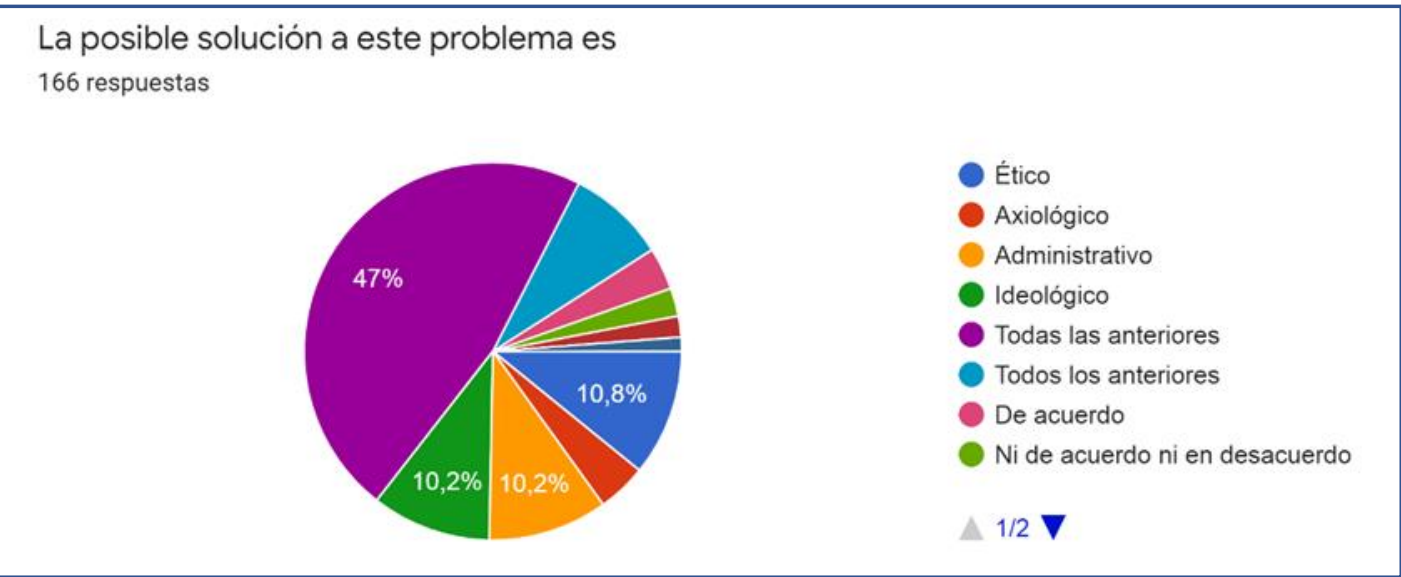

Figura 8. Características que propician la tolerancia ideológica Elaboración propia (2020).

La solución es cuestión de un aspecto ético, y los guarismos aquí son: 10,8\%, de formación ideológica, 10,2\%, de carácter administrativo. Mientras que un 47\% señala que la solución contempla todas las anteriores posibilidades. A continuación, se exponen algunos de los principales factores de la exclusión educativa registrados en este informe a partir del foco de discusión:

Cuadro $\mathrm{N}^{\circ}$ 1:

Modelo de factores de exclusión educativa

\begin{tabular}{|c|c|c|c|c|}
\hline & \multicolumn{4}{|c|}{ Niveles de intervención } \\
\hline & & Política & Institución & Salón de clase \\
\hline \multirow{3}{*}{ 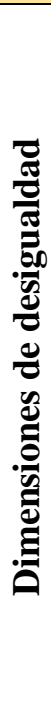 } & $\begin{array}{c}\text { Desigualdad } \\
\text { Económica } \\
\text { (redistribución) }\end{array}$ & $\begin{array}{l}\text {-Falta e inversión pública, gratuidad } \\
\text { para el acceso y oferta pública de } \\
\text { calidad } \\
\text {-planificación que prevalece ante las } \\
\text { ofertas del mercado, frente a la } \\
\text { garantía de la igualdad de } \\
\text { oportunidades } \\
\text {-Forma desegregación escolar que } \\
\text { genera una desigual distribución del } \\
\text { estudiantado }\end{array}$ & $\begin{array}{l}\text {-Prácticas de selección adversa } \\
\text { al estudiantado } \\
\text {-Distribución desigual de } \\
\text { oportunidades de éxito } \\
\text { educativo }\end{array}$ & $\begin{array}{l}\text {-Distribución desigual de recursos } \\
\text { (humanos, ocasionales, de } \\
\text { aprendizaje }\end{array}$ \\
\hline & $\begin{array}{c}\text { Desigualdad } \\
\text { cultural } \\
\text { (reconocimiento) }\end{array}$ & $\begin{array}{l}\text {-Definición limitada del concepto de } \\
\text { diversidad } \\
\text {-Falta de reconocimiento de la } \\
\text { multidimensionalidad de los procesos } \\
\text { de éxito y fracaso escolar } \\
\text {-Falta de reconocimiento social e } \\
\text { institucional de ciertas profesiones }\end{array}$ & $\begin{array}{l}\text {-Elevado academicismo que } \\
\text { no siempre reconoce los } \\
\text { cambios del entorno } \\
\text {-Desarrollo de modelos } \\
\text { simplificados de atención a la } \\
\text { diversidad } \\
\text {-Modelo de agrupación } \\
\text { homogénea del estudiantado }\end{array}$ & $\begin{array}{l}\text {-Falta de personalización del } \\
\text { proceso de aprendizaje } \\
\text {-Falta de reconocimiento de los } \\
\text { aspectos sociales y emocionales } \\
\text {-Rigidez en la organización del } \\
\text { currículo y en las prácticas } \\
\text { pedagógicas }\end{array}$ \\
\hline & $\begin{array}{c}\text { Desigualdad } \\
\text { Político }\end{array}$ & & $\begin{array}{l}\text {-Baja apertura de la institución } \\
\text { a su contexto social }\end{array}$ & \\
\hline
\end{tabular}




\begin{tabular}{|c|c|c|c|}
\hline (representación & $\begin{array}{l}\text {-Falta de participación de los } \\
\text { diferentes agentes educativos en la } \\
\text { definición de la política educativa } \\
\text {-Marco regulador centralizador que } \\
\text { limita la participación } \\
\text {-Ausencia de políticas que limitan la } \\
\text { participación }\end{array}$ & $\begin{array}{l}\text {-Falta de cultura participativa } \\
\text { en las representaciones } \\
\text { estudiantiles } \\
\text {-Baja capacidad de incidencia } \\
\text { y decisión de las familias y el } \\
\text { estudiantado }\end{array}$ & $\begin{array}{l}\text {-Modelos curriculares y } \\
\text { pedagógicos altamente } \\
\text { jerarquizados y fragmentados } \\
\text {-Debilidad del vínculo entre } \\
\text { profesorado y familias }\end{array}$ \\
\hline $\begin{array}{l}\text { Desigualdad } \\
\text { afectiva } \\
\text { (relación) }\end{array}$ & $\begin{array}{l}\text {-Falta de acompañamiento y apoyo } \\
\text { del profesorado sobre todo en las } \\
\text { acciones educativas } \\
\text {-Falta de reconocimiento del rol de las } \\
\text { emociones en los procesos de } \\
\text { enseñanza y aprendizaje }\end{array}$ & $\begin{array}{l}\text {-Prácticas de agrupación del } \\
\text { estudiantado basado en } \\
\text { expectativas sesgadas } \\
\text {-Práctica de fomento activo } \\
\text { del abandono } \\
\text {-Criterio de distribución del } \\
\text { estudiantado a grupos de } \\
\text { apoyo en base al mérito y no al } \\
\text { derecho }\end{array}$ & $\begin{array}{l}\text {-Individualización y } \\
\text { patologización del fracaso y la } \\
\text { deserción escolar } \\
\text {-Culpabilización del estudiantado } \\
\text { y familias por sus dificultades } \\
\text { educativas } \\
\text {-Ausencia de relaciones cercanas y } \\
\text { valoraciones positivas entre } \\
\text { estudiantado y profesorado }\end{array}$ \\
\hline
\end{tabular}

En resumen, se trata de un modelo holístico que permite identificar la multidimensionalidad de las formas de exclusión educativa, aunque, en función del contexto sociocultural pueden expresarse de manera diferente.

\section{Conclusión}

Se concluye desde el convencimiento de que la diversidad constituye algo "natural" e inherente a cualquier grupo humano, por lo que todo el estudiantado es diverso desde esa perspectiva. Haciendo referencia en concreto a la diversidad cultural, este estudio enfoca el trabajo en el aula reconociendo que la diversidad está vinculada a los siguientes factores.

Diferentes tipos de inteligencias y pensamientos. Es esta una característica importante que, si bien puede asociarse a espacios individuales, también se vincula a las culturas y etnias propias de cada grupo. De no tenerse en cuenta esta asociación es más probable que el estudiantado tienda a presentar un mayor rendimiento cuando su estilo de pensamiento, tipo de inteligencia prevalente no coincide con el que se potencia en la institución.

Diferentes experiencias previas culturales. Estas se adquieren en el seno de la familia y de la comunidad cercana en la que se insertan los universitarios.

Diferentes capacidades e intereses. Son propias de la diversidad real de un grupo humano, incluso dentro de una misma cultura.

La respuesta a la diversidad supone criterios como la diversificación y la flexibilidad en materiales, recursos, organización del tiempo, del trabajo y la estructuración de situaciones de enseñanza/aprendizaje que posibiliten, en el marco de una etapa determinada, que el 
mayor número de estudiantes alcance las metas propuestas, aunque sea por vías diferentes. En resumen, se trata de reconocer la diversidad como riqueza, dentro de una dinámica que aprovecha los saberes y estilos diferentes de pensar de las personas pertenecientes a diferentes culturas. 


\section{Referencias bibliográficas}

Asamblea General de las Naciones Unidas. (2006). "Convención Sobre Los Derechos De Las Personas Con Discapacidad” Articulo 24. Recuperado de: https://www.un.org/esa/socdev/enable/documents/tccconvs.pdf.

Congreso de la República de Colombia, Ministerio del Interior. (2014). Decreto 1953 de 2014 Por la cual se dictan medidas sobre Territorios Indígenas. Bogotá. Colombia.

Congreso de la República de Colombia, Ministerio del Interior. (2011). LEY 1448 DE 2011 Por la cual se dictan medidas de atención, asistencia y reparación integral a las víctimas del conflicto armado. Bogotá. Colombia.

EBERSOLD, S., \& Meijer, C. (2016). Financing Inclusive Education: Policy Challenges, Issues and Trends', Implementing Inclusive Education: Issues in Bridging the Policy-Practice Gap (International Perspectives on Inclusive Education, Volume 8).

FORLIN, C., \& Loreman, T. (Eds.). (2014). Measuring inclusive education. Emerald Group Publishing.

LÓPEZ, J. F. G. (1991). Caminando hacia el siglo XXI; la integración escolar: actas de las VII Jornadas de Universidades y Educación Especial. Universidad de Málaga.

MERCADO, O. \& De la Hoz, R. (2019). La Universidad del Atlántico, una universidad en y para la diversidad. Revista de Ciencias de la Educación, Docencia, Investigación y Tecnologías de la Información CEDOTIC., 4. (1),265-28. Disponible en: http://investigaciones.uniatlantico.edu.co/revistas/index.php/CEDOTIC/article/view/2241/2783

Ministerio de Nacional de Educación (2014). Lineamientos de política de educación superior inclusiva. Bogotá. Colombia.

OCAMPO, A. (2019). Educación Inclusiva: una reflexión aproximada sobre la formación del método. Revista de Ciencias de la Educación, Docencia, Investigación y Tecnologías de la Información CEDOTIC., 4(2), 26-51. Disponible: http://investigaciones.uniatlantico.edu.co/revistas/index.php/CEDOTIC/article/view/2281/2 922

Organización de las Naciones Unidas Para la Educación, la Ciencia y la Cultura (UNESCO). (2008). "La Educación Inclusiva: El Camino Hacia El Futuro". Cuadragésima octava reunión - Centro Internacional de Conferencias. Ginebra, Suiza: Presentación General de la $48^{a}$ reunión de la CIE. Recuperado de: http://www.ibe.unesco.org/fileadmin/user_upload/Policy_Dialogue/48th_ICE/General_Pres entation-48CIE-4_Spanish_.pdf. 
PORTER, G, y Smith, D. (2011). "Exploring Inclusive Educational Practices Through Professional Inquiry". The Netherlands: Sense Publishers. Sense Publishers. Disponible en: https://www.questia.com/read/1G1-323659506/porter-gordon-deidre-smith-eds-2011exploring

SOTO-Molina, J. (2015). Filosofía de la Liberación Intercultural y Teoría Sociológica de la Práctica: Dos Fundamentos en la Construcción de Comunidades Bilingües. Amauta, 13(26), 111-128. Disponible en: file:///C:/Users/Usuario/Desktop/DialnetFilosofiaDeLaLiberacionInterculturalYTeoriaSociolo-5440960.pdf

SOTO-Molina, J. (2017). Desde una ontología del lenguaje hacia una ética intercultural de la alteridad. Revista Amauta, 15(30), 135-150. Universidad del Atlántico. Disponible en: file:///C:/Users/Usuario/Desktop/Dialnet-DesdeUnaOntologiaDelLenguajeHaciaUnaEticalntercult6153965.pdf 